\title{
Knowledge and Perception of Plastic Surgery Among Medical Students in the Western Region of Saudi Arabia
}

\author{
Fahad Aljindan ${ }^{1}$, Ghaida Zahrani ${ }^{2}$, Nada Almalki ${ }^{3}$, Noura Alhussaini ${ }^{2}$, Nasser Aldosari ${ }^{4}$, Reema Althubaiti ${ }^{3}$, Alanoud Alsubhi ${ }^{2}$ \\ ${ }^{1}$ Department of Plastic Surgery, King Abdullah Medical City Specialist Hospital, Mecca, Saudi Arabia \\ ${ }^{2}$ College of Medicine, Ibn Sina National College, Jeddah, Saudi Arabia \\ ${ }^{3}$ College of Medicine, Taif University, Taif, Saudi Arabia \\ ${ }^{4}$ College of Medicine, Almaarefa University, Riyadh, Saudi Arabia
}

Correspondence should be addressed to Fahad Aljindan, Department of Plastic Surgery, King Abdullah Medical City Specialist Hospital, Mecca, Saudi Arabia, Email: F.aljindan@kamc.med.sa

Received: 22 September 2021, Accepted: 9 October 2021, Published: 14 October 2021

Copyright (C) 2021 Aljindan et al. This is an open access article distributed under the Creative Commons Attribution License, which permits unrestricted use, distribution, and reproduction in any medium, provided the original work is properly cited.

\begin{abstract}
Background: The future career choices of medical students are influenced by the medical school curriculum and exposure they receive in the schools. A thorough understanding of the students' knowledge and perception about a specialty like plastic surgery is essential for assessing the scope and career choices. This study evaluates the medical students' knowledge and perception of plastic surgery in the western region of Saudi Arabia.

Methods: This is a cross-sectional online survey study that was conducted using a pre-tested questionnaire which was distributed between senior medical students. The questionnaire recorded participants' sociodemographic details, their knowledge about the specialty of plastic surgery, and their perception of the specialty. The data collected were subjected to Statistical analysis using SPSS Ver.23 and a p-value of $<0.05$ was deemed significant.

Results: The majority of the students (78.3\%) reported poor knowledge regarding plastic surgery while $11 \%$ of the respondents had previously taken a clinical rotation in plastic surgery department. Female medical students had comparatively demonstrated better knowledge than male students regarding the specialty $(\mathrm{p}<0.034)$. It was reported by $43.2 \%$ of students that plastic surgery is the same as other specialties, whereas $53.2 \%$ believed that this specialty is an essential one. Only $34.1 \%$ of the students were interested in pursuing plastic surgery as a specialty after graduation.

Conclusion: The study finding highlights that the knowledge about plastic surgery is not satisfactory among medical students. There is a need to improve knowledge about this specialty. The medical curriculum should fill the voids and give more considerations for this specialty when training undergraduate medical students, which would positively influence their career choices.
\end{abstract}

Keywords: perception, western region, saudi arabia, knowledge, plastic surgery, medical students. 


\section{Journal of Healthcare Sciences}

\section{Introduction}

Around the world, plastic surgery has gained fame in the last two decades. Plastic and reconstructive surgery is a specialty that deals with the treatment of parental or acquired tissue abnormalities to improve the appearance and function of different parts of the body $(1,2)$. It is a flexible discipline in which surgeons conduct several various cosmetic and reconstructive procedures (3). Some primary care doctors, nurses, medical students, and the public appear to have very little knowledge of plastic surgery and a bad view of it (4). Plastic surgery is a wide, dynamic, and developing specialty field not limited by anatomy or organ system. Many sub-specialties are available within plastic surgery domain, including breast surgery, hand surgery, pediatric plastic surgery, skin cancer, craniofacial trauma, trauma reconstruction, oncology reconstruction of ophthalmic eye surgery and others $(5,6)$.

Over the last few years, the number of cosmetic procedures has increased, whether surgical or non-surgical (7). A Recent cross-sectional 2019 study in Saudi Arabia has shown that medical students are not sufficiently aware of plastic surgery (8). One further questionnaire-based study in India in 2017 found that their medical associates misunderstand plastic surgery as a specialty (9). A Survey study was published in 2017 , found that the perception among medical students in their study showed a gap in plastic surgery awareness (4). In 2019 research assessing the efficacy of plastic surgery simulation training for undergraduate medical students found that the experience of students Showed an improvement of $19.9 \%$ after attending the course (10). Surveys conducted in 2015 among undergraduate medical students showed that 280 students claimed that their medical education did not provide a basic understanding of specific patient problem handled by plastic surgeons (11).

The Kingdom of Saudi Arabia (KSA) ranked 30th in the top 30 countries with the highest levels of cosmetic procedures in the world according to a survey conducted by the International Society of Aesthetic Plastic Surgeons (ISAPS) in 2018 (12). According to the ISAPS, overall procedures are rising by $5.4 \%$ relative to 2017 in comparison to 2018 (12). Also, women tend to be exposed to more cosmetic procedures than men, at about $87.4 \%$, comprised to $12.6 \%$ in men of all procedures (12).

In 2015 a study was done in King Abdulaziz University (KAU) to assess the experiences and attitudes of female university students towards cosmetic surgery and reported that $2.2 \%$ received cosmetic surgery while $11.4 \%$ were potential cosmetic surgery candidates (13). In 2018, a study was done in King Khalid University to find out the knowledge of the medical students on plastic surgery and its relationship with their cultural factors. The study found that the perception towards plastic surgery was positive for $45.6 \%$ of the participants and $18.4 \%$ reported that plastic surgery is a good specialty and $34.9 \%$ reported that their biggest concerns about joining to cosmetic surgery is the difficulty to be accepted in this specialty. The study found a significant relationship between plastic surgery and cultural factors and, due to cultural factors, a large proportion of them reject plastic surgeons (14).

A recent study was conducted at Jeddah University to determine the causes of plastic surgery misperceptions among medical students and interns. The study showed that the recommendation from family or friends was the most relevant source of evidence to select plastic surgery and their greatest concern is difficult to consider in the plastic surgery program. The participants selected religious doubt as the view of the plastic surgery community in Saudi Arabia. The study concluded that the curriculum of the medical college should pay more attention to plastic surgery and concentrate on the specialty's non-cosmetic portion (15). To our knowledge, studies assessing the gap of knowledge about plastic surgery among medical students are scarce. Thus, the aim of this study was to assess knowledge and perception of plastic surgery among Medical Students in the Western Region of Saudi Arabia.

\section{Methodology}

This is a cross-sectional study which was conducted between January 2021 and July 2021. The study was done through an online survey which was distributed through social media groups among the communities of medical students. We included all male and female undergraduate medical students between third and sixth year of medical schools from the western region of Saudi Arabia. Meanwhile, we excluded first year medical students, second year medical students, interns and incomplete submissions. A predesigned questionnaire was distributed online that included 25 questions divided into three main domains. The first was participants' demographics that included age, gender, region, place of residence, university, educational level, academic GPA and nationality. The second domain was to assess their knowledge regarding the specialty of plastic surgery, and their perception about the specialty. That included areas of self- evaluation, interest in rotation, concern, cosmetic impression, the importance of plastic department, the 
Journal of Healthcare Sciences

source of information and the financial and risk issues. The third section will contain three case scenarios to assess the knowledge regarding specific plastic surgery procedures. The study was approved by the research ethics committee of the directorate of health affairs. Data were analyzed using Statistical Package for the Social Sciences (SPSS) version 25. Qualitative data was expressed as numbers and percentages, and Chi- squared test $(\chi 2)$ was applied to test the relationship between variables. A p-value of $<0.05$ was considered as statistically significant.

\section{Results}

A total of 387 student answered the survey. Out of them, $65.4 \%$ were females, $94.6 \%$ were aged $20-25$ years, $94.3 \%$ were Saudi and $45.7 \%$ were from Jeddah region. Around $29.7 \%$ from the participants studied at Taif University and $50.4 \%$ were in the 6th medical grade (Table 1). Furthermore, most of the students $(78.3 \%)$ rated their knowledge about plastic surgery as "little" and $11.1 \%$ took a plastic surgery rotation. Regarding the respondent's knowledge regarding conditions treated by plastic surgery, burns were the most commonly reported conditions chosen that are treated by plastic surgery (71.1\%). Meanwhile, the most common source of information regarding plastic surgery was the internet (59.6\%) (Table 2). Regarding the relationship students' gender and academic level, female participants were more likely to know about plastic surgery in comparison to male students $(\mathrm{p}$-value $=0.034$ ). However, there was no significant relationship between gender and academic level and their knowledge level $(p$-value $=0.384)($ Table 3).

Most students (61.7\%) responded that maxillofacial surgery was the specialty pf choice that should repair jaw fracture and cleft lip. As for the case scenario of the brachial plexus injury, $77.3 \%, 52.6 \%, 18.9 \%$ and $77.3 \%$ replied that the responsible specialties are neurosurgery, orthopedic surgery and plastic surgery, respectively. Most of the participants and replied that the specialties responsible for breast reconstruction are plastic surgery $(88.3 \%)$ and general surgery $(61.7 \%)$. However, about $78 \%$ of the students stated that specialty of choice to perform surgical tendon repairs is orthopaedic while $55.3 \%$ stated it was plastic surgery. More than half (57.8\% and $51.4 \%)$ replied correctly that the specialties responsible for face lift are plastic surgery and maxillofacial surgery respectively, while only $20.1 \%$ reported that it is the otolaryngology specialty. As for the specialty responsible for management of a second degree burn on leg, $51.1 \%$ of the participants reported that it is plastic surgery (Table 4). Almost half of the respondents $(53.2 \%)$ reported that plastic surgery is an

\begin{tabular}{|c|c|}
\hline \multicolumn{2}{|c|}{$\begin{array}{l}\text { Table 1: Distribution of studied students } \\
\text { according to their characters, college, } \\
\text { academic year and GPA }(\mathrm{N}=387)\end{array}$} \\
\hline Variable & No. $(\%)$ \\
\hline \multicolumn{2}{|l|}{ Gender } \\
\hline $\begin{array}{l}\text { Female } \\
\text { Male }\end{array}$ & $\begin{array}{l}253(65.4 \%) \\
134(34.6 \%)\end{array}$ \\
\hline \multicolumn{2}{|l|}{ Age } \\
\hline $\begin{array}{l}<20 \\
20-25 \\
26-30 \\
>30\end{array}$ & $\begin{array}{c}7(1.8 \%) \\
366(94.6 \%) \\
13(3.4 \%) \\
1(0.3 \%)\end{array}$ \\
\hline \multicolumn{2}{|l|}{ Nationality } \\
\hline $\begin{array}{l}\text { Non-Saudi } \\
\text { Saudi }\end{array}$ & $\begin{array}{c}22(5.7 \%) \\
365(94.3 \%)\end{array}$ \\
\hline \multicolumn{2}{|c|}{ Place of residence in Western Region } \\
\hline $\begin{array}{l}\text { Al Madinah } \\
\text { Jeddah } \\
\text { Makkah } \\
\text { Other } \\
\text { Taif }\end{array}$ & $\begin{array}{c}58(15 \%) \\
177(45.7 \%) \\
38(9.8 \%) \\
5(1.3 \%) \\
109(28.2 \%)\end{array}$ \\
\hline \multicolumn{2}{|l|}{ College } \\
\hline $\begin{array}{l}\text { Batterjee Medical College } \\
\text { Fakeeh College of Medical Sciences } \\
\text { Ibn Sina National College } \\
\text { Jeddah University } \\
\text { King Abdulaziz University } \\
\text { King Saud Bin Abdulaziz University } \\
\text { Other } \\
\text { Taibah University } \\
\text { Taif University } \\
\text { Umm Al Qura University }\end{array}$ & $\begin{array}{c}5(1.3 \%) \\
25(5.6 \%) \\
89(23 \%) \\
10(2.6 \%) \\
27(7 \%) \\
24(6.2 \%) \\
2(0.5 \%) \\
58(15 \%) \\
115(29.7 \%) \\
32(8.3 \%)\end{array}$ \\
\hline \multicolumn{2}{|l|}{ Academic year } \\
\hline $\begin{array}{l}3 \text { rd year } \\
4 \text { th year } \\
5 \text { th year } \\
6 \text { th year }\end{array}$ & $\begin{array}{c}39(10.1 \%) \\
73(18.9 \%) \\
80(20.7 \%) \\
195(50.4 \%)\end{array}$ \\
\hline \multicolumn{2}{|l|}{ GPA } \\
\hline $\begin{array}{l}\text { Out of } 4 \\
\text { Out of } 5\end{array}$ & $\begin{array}{l}155(40.1 \%) \\
232(59.9 \%)\end{array}$ \\
\hline \multicolumn{2}{|c|}{ GPA Grade out of 4} \\
\hline $\begin{array}{l}<2 \\
2-2.49 \\
2.5-2.99 \\
3-3.49 \\
3.5-4 \\
4.5-5\end{array}$ & $\begin{array}{c}4(1 \%) \\
4(1 \%) \\
20(5.2 \%) \\
60(15.5 \%) \\
66(17.1 \%) \\
1(0.3 \%)\end{array}$ \\
\hline \multicolumn{2}{|c|}{ GPA Grade out of 5} \\
\hline $\begin{array}{l}3-3.49 \\
3.5-3.99 \\
4-4.49 \\
4.5-5\end{array}$ & $\begin{array}{c}10(2.6 \%) \\
39(10.1 \%) \\
89(23 \%) \\
94(24.3 \%)\end{array}$ \\
\hline
\end{tabular}

* GPA: grade point average 


\section{Journal of Healthcare Sciences}

Table 2: Distribution of studied students according to their knowledge about plastic surgery $(\mathrm{N}=387)$

Variable

No. $(\%)$

How is your knowledge about plastic surgery?

Excellent

Little

$17(4.4 \%)$

$303(78.3 \%)$

No knowledge at all

$67(17.3 \%)$

The following conditions are treated by plastic surgery (you can choose more than one)

\begin{tabular}{l|c} 
Burns & $275(71.1 \%)$ \\
Rhinoplasty & $256(66.2 \%)$ \\
Breast reduction or enhancement surgeries & $265(68.5 \%)$ \\
Cleft lip and palate (Congenital) & $237(61.3 \%)$ \\
Eyelid tears and cuts over the face & $214(55.3 \%)$ \\
Congenital anomalies of ear and nose & $202(52.4 \%)$ \\
Abdominoplasty (tummy tuck) & $184(47.8 \%)$ \\
Fracture of the jaw and face & $170(44.1 \%)$ \\
Finger amputations & $87(22.7 \%)$ \\
Bedsores & $54(14.2 \%)$ \\
Maxillofacial surgery & $53(13.7 \%)$ \\
Injuries to nerve of the hand and legs & $43(11.3 \%)$ \\
Colorectal surgery & $31(8.1 \%)$ \\
Total knee replacement & $4(1 \%)$ \\
Glaucoma surgery & $2(0.5 \%)$
\end{tabular}

Have you ever taken a plastic surgery rotation?

\begin{tabular}{|l|l} 
No & $344(88.9 \%)$ \\
\hline
\end{tabular}

Yes

$43(11.1 \%)$

The following conditions are treated by plastic surgery (you can choose more than one)

Burns

$275(71.1 \%)$

Rhinoplasty

Breast reduction or enhancement surgeries

Cleft lip and palate (Congenital)

Eyelid tears and cuts over the face

Congenital anomalies of ear and nose

Abdominoplasty (tummy tuck)

Fracture of the jaw and face

Finger amputations

Bedsores

Maxillofacial surgery

Injuries to nerve of the hand and legs

Colorectal surgery

Total knee replacement

$256(66.2 \%)$

$265(68.5 \%)$

$237(61.3 \%)$

$214(55.3 \%)$

$202(52.4 \%)$

$184(47.8 \%)$

$170(44.1 \%)$

$87(22.7 \%)$

$54(14.2 \%)$

$53(13.7 \%)$

$43(11.3 \%)$

$31(8.1 \%)$

$4(1 \%)$

$2(0.5 \%)$

Glaucoma surgery

What are the sources of information regarding plastic surgery?

Internet

$230(59.6 \%)$

Television

$174(45.1 \%)$

$138(35.8 \%)$

$82(21.2 \%)$

Personal encounter

Teaching session

Clinical rotation

Workplace

Books

Others

$54(14 \%)$

$39(10.3 \%)$

$35(9.1 \%)$

$11(2.8 \%)$

$9(2.3 \%)$ essential specialty, $34.1 \%$ were interested in plastic surgery, and being competitive specialty was the biggest concern about being a plastic surgeon for $38.8 \%$ of students. About one third of students $(30.5 \%)$ thought that plastic surgery and cosmetic surgery are the same and $22.5 \%$ reported that plastic surgery procedure is scarless. Only $9.8 \%$ of students reported that they think that plastic surgery is expensive and meant for rich and famous and $68.2 \%$ reported that plastic and cosmetic surgeries has a risk that is similar as in other surgeries and very risky respectively (Table 5).

Regarding the relationship between students' gender and their perception about plastic surgery, a high number of female students reported that their biggest concern about being a plastic surgeon is being a competitive specialty $(P$-value $=0.001)$. Similarly, those who thought that plastic surgery is expensive and is meant for rich and famous was statistically significant when compared to male students (p-value $=0.04)$ (Table 6). Furthermore, when comparing age and perception of plastic surgery, students with an aged 26- 30 years had a significant higher percentage of those who reported that plastic surgery is an essential (P-value $=$ 0.028 ) In addition, those who thought that plastic surgeries are scarless was also higher in those aged 26-30 compared to other age groups $(\mathrm{p}$-value $=0.045)($ Table 7$)$.

Table 8 demonstrated that students in the sixth academic year had a significantly higher percentage of those whose biggest concern was the fact that plastic surgery is a competitive specialty, who thought that plastic surgery is expensive and that it is meant for the rich and famous and who thought that plastic and cosmetic surgeries are very risky with p-values of $0.008,0.025$ and 0.001 , respectively. Moreover, upon exploring the relationship between the student's GPA and their perception of plastic surgery, those with a GPA out of 5 had a significant higher percentage of those whose biggest concern was that plastic surgery is a competitive specialty $(\mathrm{p}$-value $=0.022)($ Table 9$)$.

Students at Jeddah University had a significantly higher percent of those who were interested in plastic surgery compared to students from other universities (p-value $<$ 0.001) (Figure 1). Students at King Saud Bin Abdulaziz University had a significantly higher percentage of those whose biggest concern about being a plastic surgeon is that it is a competitive specialty (p-value $<0.001$ ) (Figure 2).

Students at Ibn Sina National College had a significantly higher percentage compared to those who thought that plastic surgery and cosmetic surgery are the same and who thought that they are very risky (p-value $<0.001$ ) (Figure 3, 4).

* Statistically significant 


\section{Journal of Healthcare Sciences}

\begin{tabular}{|c|c|c|c|c|c|}
\hline \multirow{2}{*}{ Variable } & \multicolumn{3}{|c|}{ How is your knowledge about plastic surgery? } & \multirow{2}{*}{$\chi^{2}$} & \multirow{2}{*}{ p-value } \\
\hline & Excellent & Little & No knowledge at all & & \\
\hline \multicolumn{6}{|c|}{ Gender } \\
\hline $\begin{array}{l}\text { Female } \\
\text { Male }\end{array}$ & $\begin{array}{c}11(64.7 \%) \\
6(35.3 \%)\end{array}$ & $\begin{array}{l}189(62.4 \%) \\
114(37.6 \%)\end{array}$ & $\begin{array}{l}53(79.1 \%) \\
14(20.9 \%)\end{array}$ & 6.78 & $0.034 *$ \\
\hline \multicolumn{6}{|c|}{ Academic year } \\
\hline $\begin{array}{l}\text { 3rd year } \\
4 \text { th year } \\
5 \text { th year } \\
6 \text { th year }\end{array}$ & $\begin{array}{c}2(5.1 \%) \\
2(2.7 \%) \\
3(3.8 \%) \\
10(5.1 \%)\end{array}$ & $\begin{array}{c}31(79.5 \%) \\
53(72.6 \%) \\
60(75 \%) \\
159(81.5 \%)\end{array}$ & $\begin{array}{c}6(15.4 \%) \\
18(24.7 \%) \\
17(21.3 \%) \\
26(13.3 \%)\end{array}$ & 6.35 & 0.384 \\
\hline
\end{tabular}

Table 4: Distribution of studied students according to their response to case scenarios related to plastic surgery $(\mathrm{N}=387)$

$$
\begin{array}{l|l}
\text { Variable } & \text { No. }(\%)
\end{array}
$$

A patient has a broken jaw, what specialties should repair the jaw fracture?

Orthopedic surger

$238(61.7 \%)$

Cardiothoracic surgery

$89(23.1 \%)$

Dermatologic surgery

$6(1.8 \%)$

Plastic surgery (correct answer)

$218(56.4 \%)$

General surgery

$176(45.6 \%)$

Pediatric surgery

$10(2.6 \%)$

Gynecological surgery

$1(0.3 \%)$

Vascular surgery

$3(0.9 \%)$

Maxillofacial surgery (correct answer)

$280(72.6 \%)$

A child was born with a cleft lip. which specialties usually does the surgical repair of the cleft lip?

Orthopedic surgery

$91(23.7 \%)$

Cardiac surgery

$1(0.3 \%)$

Dermatologic surgery

$2(0.5 \%)$

Plastic surgery (correct answer)

$198(51.3 \%)$

General surgery

$130(33.6 \%)$

Pediatric surgery

Gynecological surgery

$15(3.8 \%)$

Vascular surgery

$103(26.8 \%)$

Maxillofacial surgery (correct answer)

$173(44.9 \%)$

Your patient had a significant brachial plexus injury from a motorcycle accident. What specialties treat this type of injury?

Orthopedic surgery (correct answer)

$203(52.6 \%)$

Cardiac surgery

$33(8.7 \%)$

Dermatologic surgery

$8(2.1 \%)$

Plastic surgery (correct answer)

$73(18.9 \%)$

General surgery

$129(33.5 \%)$

Pediatric surgery

$87(22.6 \%)$

Gynecological surgery

$3(0.7 \%)$

Vascular surgery

$178(46.1 \%)$

Neurosurgery (correct answer)

$298(77.2 \%)$

Friend of yours had one of her breasts removed to which specialties would you refer her to do her breast reconstruction?

Orthopedic surgery

$130(33.7 \%)$

Cardiac surgery

$28(7.4 \%)$

Dermatologic surgery

$13(3.5 \%)$

Plastic surgery (correct answer)

$341(88.3 \%)$

General surgery (correct answer)

$238(61.7 \%)$

Pediatric surgery

Gynecological surgery

$7(1.8 \%)$

Vascular surgery

$9(2.3 \%)$ 


\section{Continued. Table 4:}

A patient cut one of his hand extensor tendons. What specialties should do the tendon repair?

Orthopedic surgery (correct answer)

Cardiac surgery

Dermatologic surgery

Plastic surgery (correct answer)

General surgery

Pediatric surgery

Gynecological surgery

Vascular surgery

$304(78.6 \%)$

$11(6.6 \%)$

$16(4.1 \%)$

$214(55.3 \%)$

$146(37.8 \%)$

$45(11.6 \%)$

$0(0.0 \%)$

$66(17 \%)$

40 years women went to a play clinic reception

in order to have a face lift, which of the following specialties usually do the procedure?

Orthopedic surgery

Cardiac surgery

Dermatologic surgery

Plastic surgery (correct answer)

ENT (correct answer)

Maxillofacial surgery (correct answer)

General surgery

Pediatric surgery

Gynecological surgery

Vascular surgery

Missing data

$$
\begin{gathered}
23(5.9 \%) \\
16(4.1 \%) \\
4(1 \%) \\
224(57.8 \%) \\
78(20.1 \%) \\
199(51.4 \%) \\
98(25.3 \%) \\
37(9.5 \%) \\
0(0.0 \%) \\
9(2.3 \%) \\
17(4.4 \%)
\end{gathered}
$$

20 years young female came to the ER suffering from a second degree burn on her leg, which specialty should manage this case?

Orthopedic surgery

Cardiac surgery

Dermatologic surgery

Plastic surgery (correct answer)

General surgery

Pediatric surgery

Gynecological surgery

Vascular surgery

Missing data

$$
\begin{gathered}
19(4.9 \%) \\
7(1.8 \%) \\
94(24.2 \%) \\
198(51.1 \%) \\
153(39.5 \%) \\
11(2.8 \%) \\
0(0.0 \%) \\
38(9.8 \%) \\
17(4.4 \%)
\end{gathered}
$$

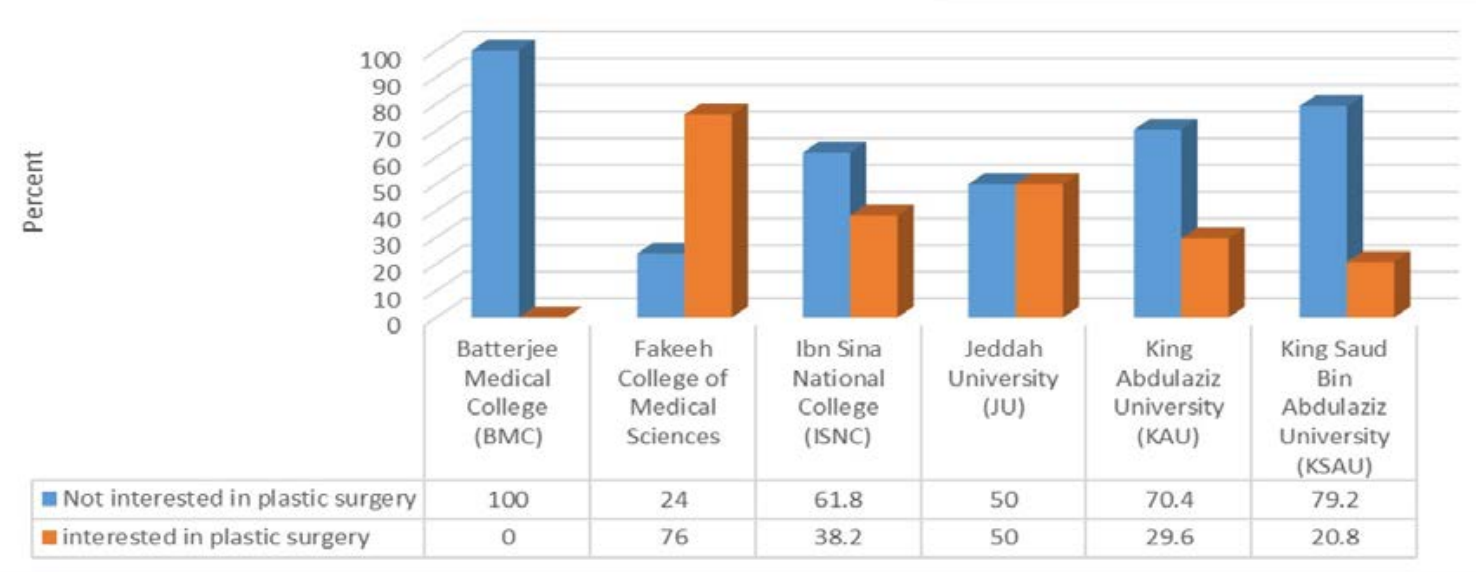

N.B.: $(\chi 2=31.08, \mathrm{p}=$ value $<0.001)$

Figure 1. Relationship between students' college and if they are interested in plastic surgery 


\section{Journal of Healthcare Sciences}

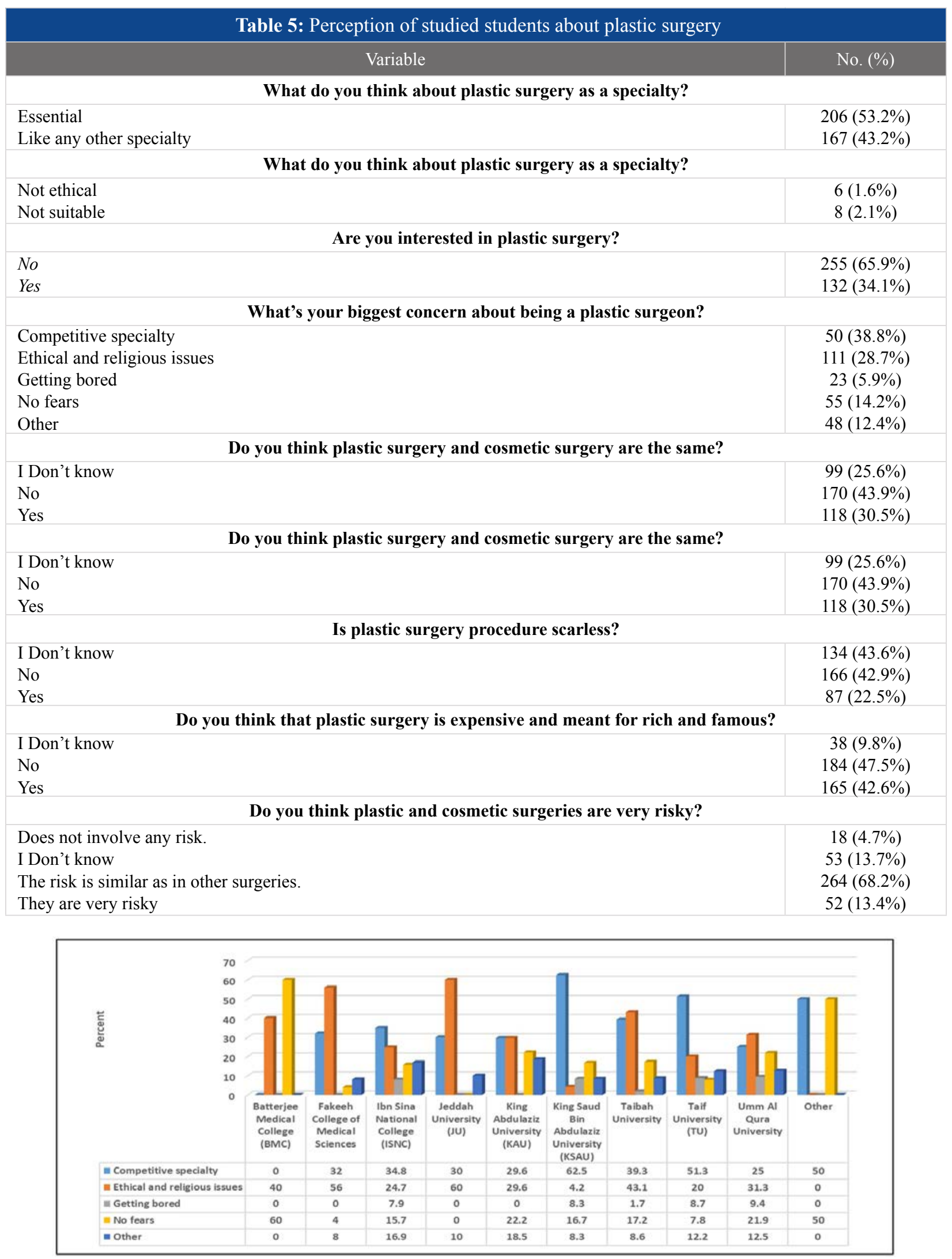

N.B.: $(\chi 2=70.19, p$-value $=0.001)$

Figure 2. Relationship between students' college and their biggest concern about being a plastic surgeon 
Journal of Healthcare Sciences

\section{Table 6: Relationship between students' gender and their perception about plastic surgery}

\begin{tabular}{|c|c|c|c|c|}
\hline \multirow[b]{2}{*}{ Variable } & \multicolumn{2}{|c|}{ Gender } & \multirow[b]{2}{*}{$\chi^{2}$} & \multirow[b]{2}{*}{ p-value } \\
\hline & $\begin{array}{l}\text { Female } \\
\text { No. }(\%)\end{array}$ & $\begin{array}{c}\text { Male } \\
\text { No. }(\%)\end{array}$ & & \\
\hline \multicolumn{5}{|c|}{ What do you think about plastic surgery as a specialty? } \\
\hline $\begin{array}{l}\text { Essential } \\
\text { Like any other specialty } \\
\text { Not ethical } \\
\text { Not suitable }\end{array}$ & $\begin{array}{c}134(65 \%) \\
109(65.3 \%) \\
4(66.7 \%) \\
6(75 \%)\end{array}$ & $\begin{array}{c}72(35 \%) \\
58(34.7 \%) \\
2(33.3 \%) \\
2(25 \%)\end{array}$ & 0.34 & 0.952 \\
\hline \multicolumn{5}{|c|}{ Are you interested in plastic surgery? } \\
\hline $\begin{array}{l}\text { No } \\
\text { Yes }\end{array}$ & $\begin{array}{c}172(67.5 \%) \\
81(61.4 \%)\end{array}$ & $\begin{array}{l}83(32.5 \%) \\
51(38.6 \%)\end{array}$ & 1.42 & 0.233 \\
\hline \multicolumn{5}{|c|}{ What's your biggest concern about being a plastic surgeon? } \\
\hline $\begin{array}{l}\text { Competitive specialty } \\
\text { Ethical and religious issues } \\
\text { Getting bored } \\
\text { No fears } \\
\text { Other }\end{array}$ & $\begin{array}{l}80(53.3) \\
86(77.5) \\
15(65.2) \\
35(63.6) \\
37(77.1)\end{array}$ & $\begin{array}{c}70(46.7) \\
25(22.5) \\
8(34.8) \\
20(36.4) \\
11(22.9)\end{array}$ & 19.77 & $0.001 *$ \\
\hline \multicolumn{5}{|c|}{ Do you think Plastic surgery and Cosmetic surgery are the same? } \\
\hline $\begin{array}{l}\text { I Don't know } \\
\text { No } \\
\text { Yes }\end{array}$ & $\begin{array}{c}61(61.6) \\
119(70) \\
73(61.9)\end{array}$ & $\begin{array}{c}38(38.4) \\
51(30) \\
45(38.1)\end{array}$ & 2.86 & 0.239 \\
\hline \multicolumn{5}{|c|}{ Is plastic surgery procedure scarless? } \\
\hline $\begin{array}{l}\text { I Don't know } \\
\text { No } \\
\text { Yes }\end{array}$ & $\begin{array}{c}92(68.7) \\
110(66.3) \\
51(58.6)\end{array}$ & $\begin{array}{l}42(31.3) \\
56(33.7) \\
36(41.4)\end{array}$ & 2.44 & 0.294 \\
\hline \multicolumn{5}{|c|}{ Do you think that plastic surgery is expensive and meant for rich and famous? } \\
\hline $\begin{array}{l}\text { I Don't know } \\
\text { No } \\
\text { Yes }\end{array}$ & $\begin{array}{c}33(86.8) \\
123(66.8) \\
97(58.8)\end{array}$ & $\begin{array}{l}5(13.2) \\
61(33.2) \\
68(41.2)\end{array}$ & 11.07 & $0.004^{*}$ \\
\hline \multicolumn{5}{|c|}{ Do you think plastic and cosmetic surgeries are very risky? } \\
\hline $\begin{array}{l}\text { Does not involve any risk. } \\
\text { I Don't know } \\
\text { The risk is similar as in other surgeries. } \\
\text { They are very risky }\end{array}$ & $\begin{array}{c}8(44.4) \\
33(62.3) \\
182(68.9) \\
30(57.7)\end{array}$ & $\begin{array}{l}10(55.6) \\
20(37.7) \\
82(31.1) \\
22(42.3)\end{array}$ & 6.54 & 0.088 \\
\hline
\end{tabular}

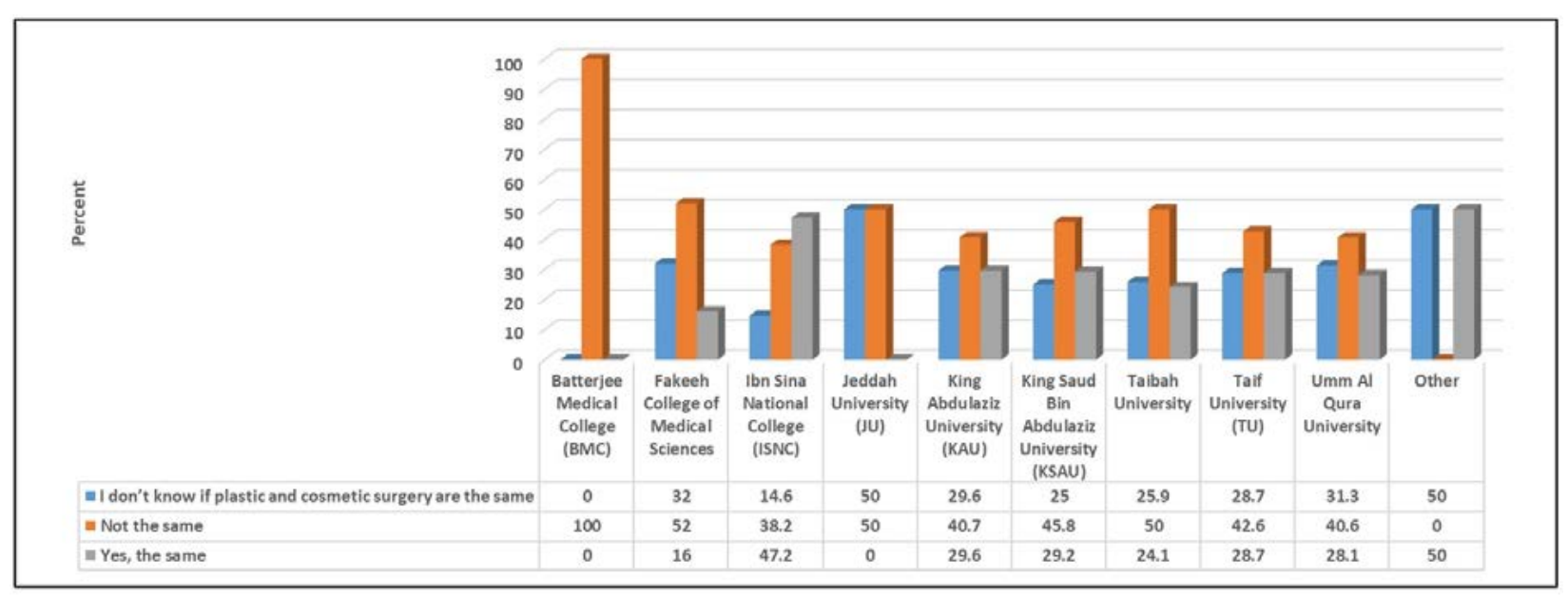

N.B.: $(\chi 2=31.6, \mathrm{p}-\mathrm{value}=0.024)$

Figure 3. Relationship between students' college and if they think plastic surgery and cosmetic surgery are the same 
Journal of Healthcare Sciences

\section{Table 7: Relationship between students' age and their perception about plastic surgery}

\begin{tabular}{|c|c|c|c|c|c|c|}
\hline \multirow[b]{2}{*}{ Variable } & \multicolumn{4}{|c|}{ Age } & \multirow[b]{2}{*}{$\chi^{2}$} & \multirow[b]{2}{*}{ p-value } \\
\hline & $\begin{array}{c}<20 \\
\text { N }(\%)\end{array}$ & $\begin{array}{l}20-25 \\
\text { N }(\%)\end{array}$ & $\begin{array}{l}26-30 \\
N(\%)\end{array}$ & $\begin{array}{c}>30 \\
\mathrm{~N}(\%)\end{array}$ & & \\
\hline
\end{tabular}

What do you think about plastic surgery as a specialty?

\begin{tabular}{|c|c|c|c|c|c|c|}
\hline $\begin{array}{l}\text { Essential } \\
\text { Like any other specialty } \\
\text { Not ethical } \\
\text { Not suitable }\end{array}$ & $\begin{array}{c}3(1.5 \%) \\
2(1.2 \%) \\
1(16.7 \%) \\
1(12.5 \%)\end{array}$ & $\begin{array}{l}0(0.0 \%) \\
1(0.6 \%) \\
0(0.0 \%) \\
0(0.0 \%)\end{array}$ & $\begin{array}{c}194(94.2 \%) \\
161(96.4 \%) \\
5(83.3 \%) \\
6(75 \%)\end{array}$ & $\begin{array}{c}9(4.4 \%) \\
3(1.8 \%) \\
0(0.0 \%) \\
1(12.5 \%)\end{array}$ & 18.68 & $0.028 *$ \\
\hline \multicolumn{7}{|c|}{ Are you interested in plastic surgery? } \\
\hline $\begin{array}{l}\text { No } \\
\text { Yes }\end{array}$ & $\begin{array}{c}5(2 \%) \\
2(1.5 \%)\end{array}$ & $\begin{array}{l}1(0.4 \%) \\
0(0.0 \%)\end{array}$ & $\begin{array}{l}241(94.5 \%) \\
125(94.7 \%)\end{array}$ & $\begin{array}{l}8(3.1 \%) \\
5(3.8 \%)\end{array}$ & 0.72 & 0.868 \\
\hline \multicolumn{7}{|c|}{ What's your biggest concern about being a plastic surgeon? } \\
\hline $\begin{array}{l}\text { Competitive specialty } \\
\text { Ethical and religious issues } \\
\text { Getting bored } \\
\text { No fears } \\
\text { Other }\end{array}$ & $\begin{array}{c}3(2 \%) \\
4(3.6 \%) \\
0(0.0 \%) \\
0(0.0 \%) \\
0(0.0 \%)\end{array}$ & $\begin{array}{l}0(0.0 \%) \\
0(0.0 \%) \\
0(0.0 \%) \\
0(0.0 \%) \\
1(2.1 \%)\end{array}$ & $\begin{array}{l}139(92.7 \%) \\
104(93.7 \%) \\
21(91.3 \%) \\
55(100 \%) \\
47(47.9 \%)\end{array}$ & $\begin{array}{l}8(5.3 \%) \\
3(2.7 \%) \\
2(8.7 \%) \\
0(0.0 \%) \\
0(0.0 \%)\end{array}$ & 18.96 & 0.089 \\
\hline \multicolumn{7}{|c|}{ Do you think Plastic surgery and Cosmetic surgery are the same? } \\
\hline $\begin{array}{l}\text { I Don't know } \\
\text { No } \\
\text { Yes }\end{array}$ & $\begin{array}{l}0(0.0 \%) \\
4(2.4 \%) \\
3(2.5 \%)\end{array}$ & $\begin{array}{l}0(0.0 \%) \\
1(0.6 \%) \\
0(0.0 \%)\end{array}$ & $\begin{array}{c}96(97 \%) \\
159(93.5 \%) \\
111(94.1 \%)\end{array}$ & $\begin{array}{c}3(3 \%) \\
6(3.5 \%) \\
4(3.4 \%)\end{array}$ & 3.82 & 0.7 \\
\hline \multicolumn{7}{|c|}{ Is plastic surgery procedure scarless? } \\
\hline $\begin{array}{l}\text { I Don't know } \\
\text { No } \\
\text { Yes }\end{array}$ & $\begin{array}{c}4(3) \\
0(0.0) \\
3(3.4)\end{array}$ & $\begin{array}{l}0(0.0) \\
1(0.6) \\
0(0.0)\end{array}$ & $\begin{array}{c}128(95.5) \\
155(93.4) \\
83(95.4)\end{array}$ & $\begin{array}{l}2(1.5) \\
10(6) \\
1(1.1)\end{array}$ & 12.86 & $0.045 *$ \\
\hline \multicolumn{7}{|c|}{ Do you think that plastic surgery is expensive and meant for rich and famous? } \\
\hline $\begin{array}{l}\text { Yes } \\
\text { No } \\
\text { I Don't know }\end{array}$ & $\begin{array}{c}0(0.0 \%) \\
2(1.1 \%) \\
5(3 \%)\end{array}$ & $\begin{array}{l}0(0.0 \%) \\
1(0.5 \%) \\
0(0.0 \%)\end{array}$ & $\begin{array}{c}37(97.4 \%) \\
173(94 \%) \\
156(94.5 \%)\end{array}$ & $\begin{array}{l}1(2.6 \%) \\
8(4.3 \%) \\
4(2.4 \%)\end{array}$ & 4.74 & 0.577 \\
\hline \multicolumn{7}{|c|}{ Do you think plastic and cosmetic surgeries are very risky? } \\
\hline $\begin{array}{l}\text { Does not involve any risk. } \\
\text { I Don't know } \\
\text { The risk is similar as in other surgeries. } \\
\text { They are very risky }\end{array}$ & $\begin{array}{l}0(0.0 \%) \\
0(0.0 \%) \\
5(1.9 \%) \\
2(3.8 \%)\end{array}$ & $\begin{array}{l}0(0.0 \%) \\
0(0.0 \%) \\
1(0.4 \%) \\
0(0.0 \%)\end{array}$ & $\begin{array}{c}18(100 \%) \\
51(96.2 \%) \\
249(94.3 \%) \\
48(92.3 \%)\end{array}$ & $\begin{array}{l}0(0.0 \%) \\
2(3.8 \%) \\
9(3.4 \%) \\
2(3.8 \%)\end{array}$ & 3.72 & 0.929 \\
\hline
\end{tabular}

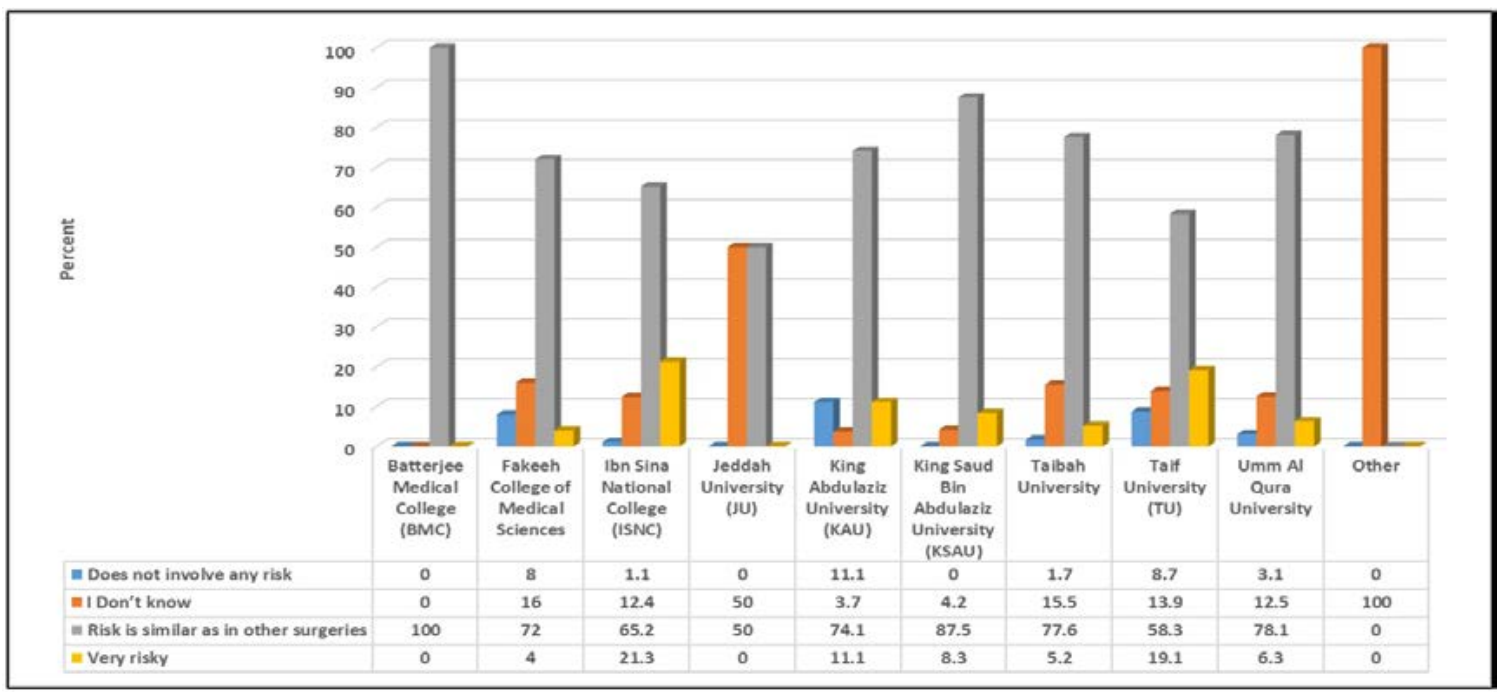

N.B.: $(\chi 2=60.4$, p-value $=<0.001)$

Figure 4. Relationship between students' college and if they think plastic and cosmetic surgeries are very risky 


\section{Journal of Healthcare Sciences}

\begin{tabular}{|c|c|c|c|c|c|c|}
\hline \multirow[b]{2}{*}{ Variable } & \multicolumn{4}{|c|}{ Academic year } & \multirow[b]{2}{*}{$\chi^{2}$} & \multirow[b]{2}{*}{ p-value } \\
\hline & $\begin{array}{l}\text { Third year } \\
\text { No. }(\%)\end{array}$ & $\begin{array}{c}\text { Fourth year } \\
\text { No. }(\%)\end{array}$ & $\begin{array}{l}\text { Fifth year } \\
\text { No. }(\%)\end{array}$ & $\begin{array}{l}\text { Sixth year } \\
\text { No. }(\%)\end{array}$ & & \\
\hline \multicolumn{7}{|c|}{ What do you think about plastic surgery as a specialty? } \\
\hline $\begin{array}{l}\text { Essential } \\
\text { Like any other specialty } \\
\text { Not ethical } \\
\text { Not suitable }\end{array}$ & $\begin{array}{l}16(7.8 \%) \\
20(12 \%) \\
1(16.7 \%) \\
2(25 \%)\end{array}$ & $\begin{array}{l}31(15 \%) \\
40(24 \%) \\
1(16.7 \%) \\
1(12.5 \%)\end{array}$ & $\begin{array}{c}46(22.3 \%) \\
28(16.8 \%) \\
3(50 \%) \\
3(37.5 \%)\end{array}$ & $\begin{array}{c}113(54.9 \%) \\
79(47.3 \%) \\
1(16.7 \%) \\
2(25 \%)\end{array}$ & 16.39 & 0.059 \\
\hline \multicolumn{7}{|c|}{ Are you interested in plastic surgery? } \\
\hline $\begin{array}{l}\text { No } \\
\text { Yes }\end{array}$ & $\begin{array}{c}23(9 \%) \\
16(12.1 \%)\end{array}$ & $\begin{array}{c}46(18 \%) \\
27(20.5 \%)\end{array}$ & $\begin{array}{l}57(22.4 \%) \\
23(17.4 \%)\end{array}$ & $\begin{array}{c}129(50.6 \%) \\
66(50 \%)\end{array}$ & 2.12 & 0.546 \\
\hline \multicolumn{7}{|c|}{ What's your biggest concern about being a plastic surgeon? } \\
\hline $\begin{array}{l}\text { Competitive specialty } \\
\text { Ethical and religious issues } \\
\text { Getting bored } \\
\text { No fears } \\
\text { Other }\end{array}$ & $\begin{array}{c}11(7.3 \%) \\
14(12.6 \%) \\
5(21.7 \%) \\
6(10.9 \%) \\
3(6.3 \%)\end{array}$ & $\begin{array}{c}23(15.3 \%) \\
25(22.5 \%) \\
2(8.7 \%) \\
16(19.1 \%) \\
7(14.6 \%)\end{array}$ & $\begin{array}{c}28(18.7 \%) \\
22(28.8 \%) \\
2(8.7 \%) \\
7(12.7 \%) \\
11(22.9 \%)\end{array}$ & $\begin{array}{l}88(58.7 \%) \\
40(36 \%) \\
14(60.9 \%) \\
26(47.3 \%) \\
27(56.3 \%)\end{array}$ & 26.99 & $0.008 *$ \\
\hline \multicolumn{7}{|c|}{ Do you think Plastic surgery and Cosmetic surgery are the same? } \\
\hline $\begin{array}{l}\text { I Don't know } \\
\text { No } \\
\text { Yes }\end{array}$ & $\begin{array}{c}15(15.2 \%) \\
18(10.6 \%) \\
6(5.1 \%)\end{array}$ & $\begin{array}{l}24(24.2 \%) \\
30(17.6 \%) \\
19(16.1 \%)\end{array}$ & $\begin{array}{l}20(20.2 \%) \\
37(21.8 \%) \\
23(19.5 \%)\end{array}$ & $\begin{array}{c}40(40.4 \%) \\
95(50 \%) \\
70(59.3 \%)\end{array}$ & 11.64 & 0.07 \\
\hline \multicolumn{7}{|c|}{ Is plastic surgery procedure scarless? } \\
\hline $\begin{array}{l}\text { I Don't know } \\
\text { No } \\
\text { Yes }\end{array}$ & $\begin{array}{c}12(9 \%) \\
14(8.4 \%) \\
13(14.9 \%)\end{array}$ & $\begin{array}{l}30(22.4 \%) \\
24(14.5 \%) \\
19(21.8 \%)\end{array}$ & $\begin{array}{l}30(22.4 \%) \\
40(24.1 \%) \\
10(11.5 \%)\end{array}$ & $\begin{array}{c}62(46.3 \%) \\
88(53 \%) \\
45(51.7 \%)\end{array}$ & 11.04 & 0.087 \\
\hline \multicolumn{7}{|c|}{ Do you think that plastic surgery is expensive and meant for rich and famous? } \\
\hline $\begin{array}{l}\text { Yes } \\
\text { No } \\
\text { I Don't know }\end{array}$ & $\begin{array}{c}0(0.0 \%) \\
21(11.4 \%) \\
18(10.9 \%)\end{array}$ & $\begin{array}{c}4(10.5 \%) \\
42(22.8 \%) \\
27(16.4 \%)\end{array}$ & $\begin{array}{c}6(15.8 \%) \\
41(22.3 \%) \\
23(20 \%)\end{array}$ & $\begin{array}{l}28(73.7 \%) \\
80(43.5 \%) \\
87(52.7 \%)\end{array}$ & 14.46 & $0.025 *$ \\
\hline \multicolumn{7}{|c|}{ Do you think plastic and cosmetic surgeries are very risky? } \\
\hline $\begin{array}{l}\text { Does not involve any risk. } \\
\text { I Don't know } \\
\text { The risk is similar as in other surgeries. } \\
\text { They are very risky }\end{array}$ & $\begin{array}{c}1(5.6 \%) \\
8(15.1 \%) \\
28(10.6 \%) \\
2(3.8 \%)\end{array}$ & $\begin{array}{l}7(38.9 \%) \\
14(26.4 \%) \\
46(17.4 \%) \\
6(11.5 \%)\end{array}$ & $\begin{array}{c}4(22.2 \%) \\
8(15.1 \%) \\
65(24.6 \%) \\
3(5.8 \%)\end{array}$ & $\begin{array}{c}6(33.3 \%) \\
23(43.4 \%) \\
125(47.3 \%) \\
41(78.8 \%)\end{array}$ & 29.75 & $0.001 *$ \\
\hline
\end{tabular}

* Statistically significant 


\section{Journal of Healthcare Sciences}

\begin{tabular}{|c|c|c|c|c|}
\hline \multirow[b]{2}{*}{ Variable } & \multicolumn{2}{|c|}{ GPA } & \multirow[b]{2}{*}{$\chi^{2}$} & \multirow[b]{2}{*}{ p-value } \\
\hline & $\begin{array}{l}\text { Out of } 4 \\
\text { No. }(\%)\end{array}$ & $\begin{array}{l}\text { Out of } 5 \\
\text { No. }(\%)\end{array}$ & & \\
\hline \multicolumn{5}{|c|}{ What do you think about plastic surgery as a specialty? } \\
\hline $\begin{array}{l}\text { Essential } \\
\text { Like any other specialty } \\
\text { Not ethical } \\
\text { Not suitable }\end{array}$ & $\begin{array}{c}81(39.3 \%) \\
68(40.7 \%) \\
2(33.3 \%) \\
4(50 \%)\end{array}$ & $\begin{array}{c}125(60.7 \%) \\
99(59.3 \%) \\
4(66.7 \%) \\
4(50 \%)\end{array}$ & 0.51 & 0.915 \\
\hline \multicolumn{5}{|c|}{ Are you interested in plastic surgery? } \\
\hline $\begin{array}{l}\text { No } \\
\text { Yes }\end{array}$ & $\begin{array}{c}103(40.4 \%) \\
52(39.4 \%)\end{array}$ & $\begin{array}{c}152(59.6 \%) \\
80(60.6 \%)\end{array}$ & 0.03 & 0.849 \\
\hline \multicolumn{5}{|c|}{ What's your biggest concern about being a plastic surgeon? } \\
\hline $\begin{array}{l}\text { Competitive specialty } \\
\text { Ethical and religious issues } \\
\text { Getting bored } \\
\text { No fears } \\
\text { Other }\end{array}$ & $\begin{array}{l}68(45.3 \%) \\
35(31.5 \%) \\
14(60.9 \%) \\
17(30.9 \%) \\
21(43.8 \%)\end{array}$ & $\begin{array}{c}82(54.7 \%) \\
76(68.5 \%) \\
9(39.1 \%) \\
38(69.1 \%) \\
27(56.3 \%)\end{array}$ & 11.43 & $0.022 *$ \\
\hline \multicolumn{5}{|c|}{ Do you think Plastic surgery and Cosmetic surgery are the same? } \\
\hline $\begin{array}{l}\text { I Don't know } \\
\text { No } \\
\text { Yes }\end{array}$ & $\begin{array}{c}45(45.5 \%) \\
68(40 \%) \\
42(35.6 \%)\end{array}$ & $\begin{array}{c}54(54.5 \%) \\
102(60 \%) \\
76(64.4 \%)\end{array}$ & 2.18 & 0.336 \\
\hline \multicolumn{5}{|c|}{ Is plastic surgery procedure scarless? } \\
\hline $\begin{array}{l}\text { I Don't know } \\
\text { No } \\
\text { Yes }\end{array}$ & $\begin{array}{l}56(41.8 \%) \\
62(37.3 \%) \\
37(42.5 \%)\end{array}$ & $\begin{array}{c}78(58.2 \%) \\
104(62.7 \%) \\
50(57.5 \%)\end{array}$ & 0.89 & 0.639 \\
\hline \multicolumn{5}{|c|}{ Do you think that plastic surgery is expensive and meant for rich and famous? } \\
\hline $\begin{array}{l}\text { I Don't know } \\
\text { No } \\
\text { Yes }\end{array}$ & $\begin{array}{l}17(44.7 \%) \\
66(35.9 \%) \\
72(43.6 \%)\end{array}$ & $\begin{array}{l}21(55.3 \%) \\
118(64.1 \%) \\
93(56.4 \%)\end{array}$ & 2.57 & 0.277 \\
\hline \multicolumn{5}{|c|}{ Do you think plastic and cosmetic surgeries are very risky? } \\
\hline $\begin{array}{l}\text { Does not involve any risk. } \\
\text { I Don't know } \\
\text { The risk is similar as in other surgeries. } \\
\text { They are very risky }\end{array}$ & $\begin{array}{l}11(61.1 \%) \\
22(41.4 \%) \\
98(37.1 \%) \\
24(46.2 \%)\end{array}$ & $\begin{array}{c}7(38.9 \%) \\
31(58.5 \%) \\
166(62.9 \%) \\
28(53.8 \%)\end{array}$ & 5.12 & 0.163 \\
\hline
\end{tabular}

* Statistically significant, GPA: grade point average

\section{Discussion}

In Saudi Arabia, there are limited training options for plastic or reconstructive surgery (16), and it would be advantageous to learn the medical students' attributes, characteristics, and other factors that influence their decision in pursuing this surgical subspecialty. Thus, our study explored the knowledge and perceptions of plastic surgery among medical students pursuing their degree in the Western region of KSA. The study findings showed that the majority of the students reported that they have little knowledge regarding this specialty. A recent study done among undergraduate male medical students in one of the universities in Saudi Arabia showed that $68 \%$ of them reported good knowledge regarding plastic surgery (14).
Approximately $89 \%$ of the students agreed that they took rotation in plastic surgery during their internship. In Saudi Arabia, to get a residency seat for plastic surgery, applicants should have above-average academic achievement and a high number of scientific publications (17). The female students showed significantly higher knowledge about this subspecialty than male students in this study. This finding is also reported in other studies done by Mortada et al. in Jeddah, Saudi Arabia (18). The possible explanation for this could be that females are more concerned about body appearance and cosmetics than males (19). This would have made female students inquire and acquire more information about plastic surgery and think of it as a career option. It may be more difficult to develop a competitive application 


\section{Journal of Healthcare Sciences}

greater interest in taking plastic surgery as a career option after graduation. This could be explained on the basis of differences between schools in exposure to plastic surgery during undergraduate medical training (20). There is a need to restructure the formal teaching methods in plastic surgery, and this should include a brief but broad overview of this specialty during the first two years of undergraduate medical training. The curriculum could include didactic lectures, workshops for skill developments, computerassisted learning, mandatory rotations in plastic surgery clinics during clinical years, or a combination of the above strategies (21). Regardless of which teaching modality is used, students should be formally assessed on what they learned as this would motivate them to transform their understanding to its application. Also, students should be encouraged to participate in case-based learning related to plastic and reconstructive surgery, which can be another effective way to improve the understanding of the specialty. One of the issues faced in this regard is that surgical specialties are facing difficulties in converting the considerations of students into genuine interest. It is reported that students who were interested in surgery during their undergraduate training were more stable in retaining their choice for residency in surgery (22). Therefore, efforts should be made by focusing on student groups who are career-malleable and inquisitive about plastic surgery, as this could lead to a higher probability of continuing on to a plastic surgery career. This is supported by other studies, which report that early exposure to surgical specialties could positively influence career choice $(23,24)$. Evidence also shows that resident mentoring and role modeling could increase the students' perceptions and career choices $(25,26)$. The study findings highlight that there is a misconception about plastic surgery among medical students as the mainstream media displays it as synonymous with cosmetic or aesthetic surgery, which could influence the attitudes of students who have not attended clinical rotations or had enough exposure to this specialty. The inclusion of plastic surgery in the undergraduate medical curriculum is also a controversia and debatable topic, and evidence shows that this specialty is underrepresented in the curriculum in most medical schools (27). This study signifies the need for the incorporation of plastic surgery into the curriculum as this does not increase the knowledge regarding the specialty but also improves the students' clinical decision-making skills.

One of the major limitations of the study is its cross-sectional approach as these involved only medical students from the western region of Saudi Arabia. Therefore, the knowledge level and perceptions are not representative of all medical students in the country. Secondly, we didn't assess the actual exposure and training that student received in their respective institutions. And finally, our findings were based on self-reported responses, which could have resulted in social desirability bias.

\section{Conclusion}

The study findings showed that the knowledge and perception about plastic surgery are not that satisfactory among medical students. The poor understanding of the specialty's significance and its future opportunities could have made most medical students not to consider it as a carrier option. Students should be more exposed to clinical rotation in plastic surgery departments for better outcomes. Resident mentoring and role modeling could also improve the students' perceptions and career choices.

\section{Disclosure}

\section{Statement:}

The authors declare no conflict of interest.

\section{Funding:}

None.

\section{Ethical Consideration:}

The study was approved by the ethical committee of directorate of health affairs in the western region with approval number A01171 and registration number H-02-J-002.

\section{Data Availability:}

The survey used in the study is found within the supplementary materials.

\section{Author Contribution:}

All authors equally contributed to conceptualizing, drafting, searching the literature, writing, language editing and proofreading the manuscript.

\section{References:}

1. Swami V, Taylor R, Carvalho C. Acceptance of cosmetic surgery and celebrity worship: Evidence of associations among female undergraduates. Personality and Individual Differences. 2009;47(8):869-72.

2.Yun S, Na Y, Jin Y, Hur E, Heo W, Lee J, et al. A Survey Study on Professional Women's Perception toward Cosmetic Surgery: 4 Year Comparison. Archives of Aesthetic Plastic Surgery. 2015;21(2):70-4. 


\section{Journal of Healthcare Sciences}

3. Park A, Scerri G, Benamore R, McDiarmid J, Lamberty B. What do plastic surgeons do? Journal of the Royal College of Surgeons of Edinburgh. 1998;43(3):189-93.

4. Fraser SJ, Al Youha S, Rasmussen PJ, Williams JG. Medical Student Perception of Plastic Surgery and the Impact of Mainstream Media. Plast Surg (Oakv). 2017;25(1):48-53.

5. Reformat DD, David JA, Diaz-Siso JR, Plana NM, Wang A, Brownstone ND, et al. How many people work in your operating room? An assessment of factors associated with instrument recounts within plastic surgery. Journal of plastic, reconstructive \& aesthetic surgery : JPRAS 2017;70(9):1285-91.

6. Chao AH, Khansa I, Kaiser C, Bell J, Miller MJ. The differential impact of plastic surgery subspecialties on the financial performance of an academic clinical practice. Plastic and reconstructive surgery. 2014;133(6):748e-55e.

7. Almasri RA, Alomawi MA, Fahad M, Alhabshan HA, Alosaimi MS. Number of cosmetic procedures among women in Saudi community. International Journal of Medicine in Developing Countries. 2019;3(11):920-5.

8. Dow T, McGuire C, Crawley E, Davies D. Application rates to surgical residency programs in Canada. Can Med Educ J. 2020;11(3):e92-e100.

9. Davis CR, O'Donoghue JM, McPhail J, Green AR. How to improve plastic surgery knowledge, skills and career interest in undergraduates in one day. Journal of plastic, reconstructive \& aesthetic surgery : JPRAS 2010;63(10):1677-81.

10. Zargaran D, Turki M, Farzaneh B, Subramaniam M, Motahariasl N, Zargaran A. Evaluating the effectiveness of plastic surgery simulation training for undergraduate medical students. Journal of plastic, reconstructive \& aesthetic surgery : JPRAS. 2020;73(2):276-7.

11. Panse N, Panse S, Kulkarni P, Dhongde R, Sahasrabudhe P. Awareness and Perception of Plastic Surgery among Healthcare Professionals in Pune, India: Do They Really Know What We Do? Plast Surg Int. 2012;2012:962169-.

\section{TO MBWY. ISAPS NEWS. 2018.}

13. Al-Saiari AA, Bakarman MA. Experiences and attitude among Saudi female University students towards cosmetic surgery. Journal of Taibah University Medical Sciences. 2015;10(4):427-31.

14. Fayi KA, Al-Sharif MN, Alobaidi AA, Alqarni MA, Alghamdi MH, Alqahatani BA. Male medical students' perception of plastic surgery and its relationship with their cultural factors. J Family Med Prim Care. 2018;7(6):1482-7.
15. Fakiha M. Mohaned Al Safar, Mohammed Alsatti, Salih Aljehany, Hisham Rizq. The causes of medical students and interns misperception of plastic surgery specialty at University of Jeddah. Medical Science. 2020;24(103):1200-7.

16. Shah Mardan QNM, Alamari NA, Alzahrani HM, Almarghoub MA, Al Saud NA, Alqahtani MS. The Ideal Applicant to the Saudi Plastic Surgery Residency Program. Plastic and Reconstructive Surgery - Global Open. 2021;9(2).

17. Program NRM. Main Residency Match Data and Reports. 2019.

18. Mortada HH, Alqahtani YA, Seraj HZ, Albishi WK, Aljaaly HA. Perception of Plastic Surgery and the Role of Media Among Medical Students: Cross-Sectional Study. Interactive journal of medical research. 2019;8(2):e12999.

19. Quittkat HL, Hartmann AS, Düsing R, Buhlmann U, Vocks S. Body Dissatisfaction, Importance of Appearance, and Body Appreciation in Men and Women Over the Lifespan. Frontiers in psychiatry. 2019;10:864.

20. Austin RE, Wanzel KR. Exposure to plastic surgery during undergraduate medical training: A single-institution review. Plast Surg (Oakv). 2015;23(1):43-7.

21. Aboelmagd S. Undergraduate plastic surgery: Is extracurricular education enough? Journal of Plastic, Reconstructive \& Aesthetic Surgery. 2010;63(2):e133.

22. Scott I, Gowans M, Wright B, Brenneis F. Stability of medical student career interest: a prospective study. Academ

23. Erzurum VZ, Obermeyer RJ, Fecher A, Thyagarajan P, Tan P, Koler AK, et al. What influences medical students' choice of surgical careers. Surgery. 2000;128(2):253-6.

24. Kozar RA, Lucci A, Miller CC, Azizzadeh A, Cocanour CS, Potts JR, et al. Brief intervention by surgeons can influence students toward a career in surgery. The Journal of surgical research. 2003;111(1):166-9.

25. Cochran A, Paukert JL, Scales EM, Neumayer LA. How medical students define surgical mentors. American journal of surgery. 2004;187(6):698-701.

26. Ravindra P, Fitzgerald JE. Defining surgical role models and their influence on career choice. World journal of surgery. 2011;35(4):704-9.

27. Coyan GN, Kilic A, Gleason TG, Schuchert MJ, Luketich JD, Okusanya $O$, et al. Medical student perceptions of a career in cardiothoracic surgery: Results of an institutional survey. The Journal of thoracic and cardiovascular surgery. 2020;159(5):1906-12. 